\title{
Coexisting With Different Human-Wildlife Coexistence Perspectives
}

\author{
Jenny Anne Glikman ${ }^{1,2 *}$, Beatrice Frank ${ }^{3,4}$, Kirstie A. Ruppert ${ }^{2}$, Jillian Knox ${ }^{2,5}$, \\ Carly C. Sponarski $i^{6}$, Elizabeth Covelli Metcalf ${ }^{7}$, Alexander L. Metcalf ${ }^{7}$ and \\ Silvio Marchini ${ }^{8,9,10}$
}

1 Instituto De Estudios Sociales Avanzados (IESA-CSIC), Córdoba, Spain, ${ }^{2}$ San Diego Zoo Wildlife Alliance, Escondido, CA, United States, ${ }^{3}$ Capital Regional District-Regional Parks Canada, Victoria, BC, Canada, ${ }^{4}$ School of Environmental Studies, University of Victoria, Victoria, BC, Canada, ${ }^{5}$ Sandra Day O'Connor College of Law, Arizona State University, Tempe, AZ, United States, ${ }^{6}$ Natural Resources Canada, Canadian Forest Service, Northern Forestry Centre, Edmonton, AB, Canada, ${ }^{7}$ Wildlife Biology Program, University of Montana Human Dimensions Lab, Missoula, MT, United States, ${ }^{8}$ Wildlife Conservation Research Unit, Department of Zoology, University of Oxford, Oxford, United Kingdom, ${ }^{9}$ Laboratório de Ecologia, Manejo e Conservação de Fauna Silvestre, Departamento de Ciências Florestais, Escola Superior de Agricultura "Luiz de Queiroz", Universidade de São Paulo, São Paulo, Brazil, ${ }^{10}$ North of England Zoological Society (Chester Zoo), Chester, United Kingdom

Over the last decade, there has been a remarkable increase in scientific literature addressing human-wildlife interactions $(\mathrm{HWI})$ and associated concepts, such as coexistence, tolerance, and acceptance. Despite increased attention, these terms are rarely defined or consistently applied across publications. Indeed, the meaning of these concepts, especially coexistence, is frequently assumed and left for the reader to interpret, making it hard to compare studies, test metrics, and build upon previous HWI research. To work toward a better understanding of these terms, we conducted two World Café sessions at international conferences in Namibia, Africa and Ontario, Canada. Here, we present the array of perspectives revealed in the workshops and build upon these results to describe the meaning of coexistence as currently applied by conservation scientists and practitioners. Although we focus on coexistence, it is imperative to understand the term in relation to tolerance and acceptance, as in many cases these latter terms are used to express, measure, or define coexistence. Drawing on these findings, we discuss whether a common definition of these terms is possible and how the conservation field might move toward clarifying and operationalizing the concept of human-wildlife coexistence.

Keywords: human-wildlife acceptance, human-wildlife conflict, human-wildlife interactions, human-wildlife tolerance, World Café, conservation lexicon

\section{INTRODUCTION}

Research on human-wildlife interactions has evolved from a focus on conflict to the inclusion of coexistence, acceptance, and tolerance (Woodroffe et al., 2005; Frank, 2016; Pooley et al., 2017, 2020; Frank et al., 2019). While various definitions and metrics of human-wildlife conflict have been proposed, tested, and applied over the last decade (Redpath et al., 2015; IUCN, 2020; Treves and Santiago-Ávila, 2020), the term coexistence is still defined and applied inconsistently throughout the relevant literature (Carter and Linnell, 2016; Chapron and López-Bao, 2016; Morehouse and Boyce, 2017). For example, Frank (2016) defines coexistence as a balance or a negotiated 
compromise between humans and wildlife on how to exist together; Chapron and López-Bao (2016) use an ecological community perspective where coexistence happens when species have different ecological niches and moderately compete for resources. The latter definition sees humans as super predators and questions whether humans can become less competitive and differentiate their niche to avoid conflict with species with overlapping needs. Further, recent scientific literature reviews show that focusing on the term coexistence alone is likely insufficient (e.g., Brenner and Metcalf, 2020; Knox et al., 2021). Thus, researchers may ascribe different meanings to coexistence, tolerance, and acceptance based on their worldviews and sociocultural, political, and economic contexts. It is imperative to understand the relationship between coexistence, tolerance, and acceptance as they are often used interchangeably, without clear definition, or to define one another (Bruskotter and Wilson, 2014; Kansky et al., 2016). This lack of shared understanding makes it difficult to compare case studies, test metrics, and build on previous research to create innovative and equitable solutions that enable humans and wildlife to share the same landscape.

To work toward a better understanding of what coexistence means, and its relationship with tolerance and acceptance, we ran two World Café sessions at international conferences; one in Namibia, Africa, at the "Pathways: Human Dimensions of Wildlife (Pathways)" conference in January 2018, and another in Ontario, Canada, at the "North American Congress for Conservation Biology (NACCB)" in July 2018. The World Café method is an engagement process that recreates an informal cafe-table setting where four or five participants discuss an issue in rounds of conversations. The facilitators (KR at Pathways and JG at NACCB) organized three progressive conversation rounds of approximately $20-30 \mathrm{~min}$ each and instructed participants about their tasks. Each table had markers and poster-sized paper, which allowed participants to doddle, draw, and visually record the collective knowledge created through their conversations (The World Café, 2015). Ethical review and approval was obtained through the Institutional Review Board (IRB\# 03849e) of Miami University, Ohio, before the world cafés and informed consent was requested verbally from participants during the sessions.

During the first round of discussions, we asked participants (i) whether these three terms were synonyms, and if not, how they relate to one another (e.g., one containing the other, the three situated along a continuum), and (ii) how these concepts could be defined. In a second round, we asked participants what conservation success looks like in terms of coexistence, tolerance, and acceptance. In the final round, we asked participants to describe the most important factors that comprise coexistence, acceptance, and tolerance with a view toward operationalizing the terms.

In this article, we present the array of perspectives shared by participants and build upon these results toward a more grounded understanding of coexistence, as applied by conservation scientists and practitioners. Drawing on these findings, we discuss what factors could help clarify and operationalize the concept of human-wildlife coexistence.

\section{RESULTS}

A total of 56 participants attended the two World Café sessions (20 in Namibia, and 36 in Ontario). As it was out the scope of the Word Café, and therefore of this perspective, we did not record participants' nationalities or backgrounds; however, we can state that a majority of the participants were academics (i.e., professors and graduate students) and/or practitioners who work mainly in Africa (Pathways conference) or worldwide (NACCB conference). In line with the conferences' themes, focus, Pathways participants predominately had social science backgrounds, whereas NACCB participants had natural science and socio-ecological backgrounds.

\section{Are Coexistence, Tolerance, and Acceptance Synonyms? If Not, Then How Do They Relate to Each Other? How Should Each of Them Be Defined?}

Participants perceived that although tolerance and acceptance were closer to each other than to coexistence, there was a high degree of similarity among all three terms. There was no consensus on how these concepts should be organized in relation to one another, though many participants positioned coexistence as connoting a more positive situation than tolerance and acceptance (Figure 1A). Compared to the other terms, tolerance was described as a more passive state with the implication of burden-a state of agreeing to disagree. Ability to influence decision-making processes was often seen as a factor affecting the level of tolerance; lack of power was described as leading to lower tolerance. Acceptance, in contrast, was described as a state in which the value of a species was recognized, yet there was no active promotion of human-wildlife coexistence-once again suggesting a more passive state than that of coexistence. The concepts of tolerance and acceptance were sometimes perceived as leading to coexistence (Figure 1A) and by some participants as at the same level of valence (Figure 1B).

Several prominent ideas emerged regarding definitions of the three concepts. Participants emphasized that although universal definitions could facilitate comparisons and evidence compilation, agreement on such definitions is likely impractical. Conservation programs operate within complex systems, so terminology must be adaptable to different contexts. For example, multiple participants described coexistence as a dynamic state composed of multiple dimensions, including spatial, temporal, social, and institutional facets. They also noted that coexistence often implies interactions between social and ecological elements of a system. In general, participants expressed that coexistence would not be bound with an endpoint, but rather, underpin harmony over time.

\section{What Does Conservation Success for Human-Wildlife Interactions Look Like? What Do we Ultimately Want to Achieve in Conservation: Coexistence, Tolerance, or Acceptance?}

Similar to the discussion around standard universal definitions, conservation success was seen by many as context-dependent. 


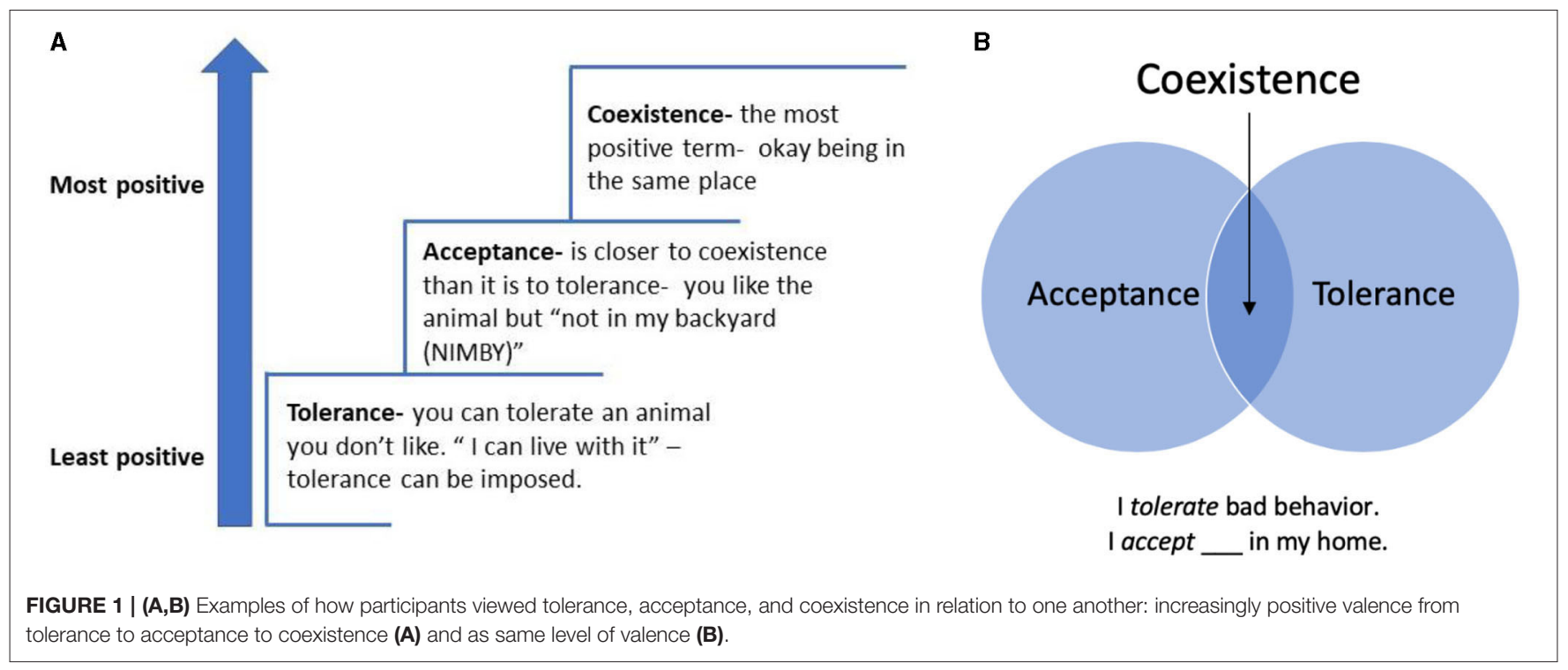

When coexistence is the end-goal, participants identified significant aspects of successful conservation, including a balance between the costs and the benefits associated with wildlife, reduced levels of conflict and negative interactions between humans and wildlife, and human behavioral changes that decrease pressure on wildlife populations. In addition to these outcome-based metrics, processes that influence success were also listed, such as productive collaboration, inclusivity, respect, and balanced power relations in conservation programs. Participants expressed differing perspectives on whether success can be labelled as coexistence, although multiple participants described coexistence as a better outcome than thresholds associated with tolerance and acceptance.

Mirroring the first round, some participants emphasized the need for flexibility - in particular, the ability to adapt definitions of success relative to coexistence, so terms reflect social outcomes (e.g., human well-being), ecological outcomes (e.g., population persistence), and resilience at relevant scales.

\section{What Factors Are Most Important to Measure Coexistence, Tolerance, and Acceptance?}

Reflecting the complexity of the three terms, participants stressed that both quantitative and qualitative measurement are valuable. Participants that conceptualized tolerance as a threshold offered behavior-based measures like the number of retaliatory killings of wildlife. Other measurable factors were attitudes, agreement regarding wildlife management strategies, and perceptions of wildlife-related costs and benefits. Participants suggested that acceptance and tolerance might be best measured at the individual level, but also may form a threshold of social carrying capacity at the collective level. Coexistence, in contrast, spans populations, ecosystems, and landscapes. Having a multiscale nature, coexistence necessitates a composite measure that captures the overlap in scales of social and ecological dimensions.
In addition, the participants identified the following relative factors of coexistence, or characteristics of the state of coexistence in some contexts: (i) benefits of existing together are equal to or outweigh the costs for both sides; (ii) negative interactions between two groups is non-existent, low, or tolerable; (iii) tolerance is high; (iv) acceptance is high; (v) neither humans or wildlife species are substantially negatively impacting the other; (vi) species are thriving; (vii) attitudes toward the species are positive or at least neutral; (viii) the state may be unstable. Coexistence is a state to strive for with clear metrics to guide goal attainment/success.

\section{DISCUSSION}

Participants found it challenging to define tolerance, acceptance, and coexistence. Instead, they supported more fluid definitions of the terms based on the contextual dimensions associated with each (i.e., spatial, temporal, social, and institutional) and the conservation issue at hand. However, despite hesitancy to strictly define these terms, many participants felt it was important to identify indicators and metrics for tolerance and acceptance, as they are not perceived as synonymous (e.g., Bruskotter and Wilson, 2014; Lute and Carter, 2020). Tolerance can be understood as having both negative and positive connotations. Specifically, it can be viewed as a virtue in that people wish to be considered tolerant. Yet being tolerant might not mean that a person prefers to assent to a specific situation; people might tolerate a situation just because they have no other option. This is similar to how Kansky et al. (2021) define tolerance: "the ability of an individual to absorb the potential or actual costs of living with wildlife" (Kansky et al., 2021, p. 604). Tolerance was seen as a lower bar than acceptance and coexistence. Acceptance was perceived as unassociated with whether people held a specific opinion or were impacted by conflict.

Coexistence was perceived as an overarching concept and participants advocated for context-specific definitions crafted 
by the local stakeholders. Overall, participants' descriptions of coexistence had commonalities despite the variation in conservation context. For example, participants felt that for coexistence to occur, the species and humans must be living in/sharing the same landscape, at the same time, which is supported by some authors (Carter and Linnell, 2016; Crespin and Simonetti, 2019), but not others (Treves and Santiago-Ávila, 2020). Participants added another characteristic to coexistence: that neither species is inhibiting the survival or sustained existence of the other species, which distinguishes coexistence from co-occurrence (Morehouse and Boyce, 2017; Lamb et al., 2020). What we found even more unique in the participants' perspectives was the scaled nature of the three terms: tolerance begets acceptance, begets coexistence (Figure 1A). This point has been debated in the literature, some indicating that for coexistence to occur, attempts should be made to increase acceptance (Lute and Carter, 2020) over tolerance (van Eeden et al., 2021). As Glikman et al. (2019) and König et al. (2020) suggest, we stress that coexistence is a dynamic process. Furthermore, a dynamic state of coexistence parallels the panarchy framework for understanding resilience in socialecological systems across scales (Holling, 2001), a perspective that embraces system dynamism through cycles of growth, conservation, release, and reorganization.

Consistent with some literature (Glikman et al., 2019; Knox et al., 2021), participants did not perceive a strong necessity to have strict definitions for the three terms. Yet, there was agreement and recommendations that these terms should be defined by the specific conservation groups working on a particular issue or conservation program (i.e., government organizations, conservation organizations). We agree that defining these terms is paramount when coexistence, tolerance, and/or acceptance are adopted as objectives of a project, with indicators and specific metrics used to guide measures of success. As previously discussed, the way we define coexistence matters (Glikman et al., 2019). The definitions we use help us understand and frame which measures, approaches, and innovations conservationists implement to promote coexistence. For example, if coexistence is defined as human and wildlife peacefully sharing landscapes, we may strive to minimize humanwildlife interactions, as avoidance of negative interaction may help maintain peace. This scenario is exemplified by cases that involve damage reduction like bear-proofing measures to reduce access to residential garbage (Johnson et al., 2018). If instead we strive for a concept of human-wildlife coexistence that entails species recovery and expansion, avoidance may not be enough and success may be reached when local communities become stewards of a species, as exemplified by Lion Guardians (Hazzah et al., 2019). We realize that the state of coexistence, like humanwildlife relationships, may be fragile and ever shifting (Yurco et al., 2017; Frank and Glikman, 2019). Whatever the details, agreeing on a definition of coexistence ahead of time can help focus efforts on the outcomes most valued by stakeholders and indicate when success is reached and/or when a definition needs to be re-assessed and adapted to a newly desired humanwildlife condition.

\section{Seeds to Operationalize Terms}

It is evident from both workshops that stakeholders should define tolerance, acceptance, and coexistence to fit their conservation contexts. This presents a challenge on how to define and measure these items across studies. The perspectives expressed in the workshops support building on a measure of tolerance for wildlife, such as that proposed by Brenner and Metcalf (2020). Specifically, further work should focus on better understanding human behavioral and attitudinal attributes toward wildlife or its behavior. Further, the workshops' results indicate that acceptance and tolerance are different and the terms should not be used as synonyms (e.g., Bruskotter et al., 2015; Slagle and Bruskotter, 2019), nor to define one another (e.g., Lischka et al., 2019). Acceptance was described as a step above tolerance, begetting coexistence, and involving recognition of the value of a species (Figure 1A). As such, potential future studies should focus on the plurality of values toward wildlife to identify attributes of acceptance. While tolerance and acceptance were generally conceptualized at the individual-level, coexistence was frequently viewed from a systems perspective, referring to a socioecological state comprised of interactions between social and ecological components. Coexistence can be humanto-animal but also human-to-human. As suggested by Pooley et al. (2020), coexistence requires a careful approach where researchers "listen carefully to and learn from others" (Pooley et al., 2020, page 06). Then, operationalizing these terms can be done a priori using closed ended questions or $a$ posteriori using open ended questions. Closed ended or Likertstyle scale questions require consideration and definition of different components of tolerance, acceptance, and coexistence. Open-ended questions allow stakeholders to define these terms for their specific context. For example, questions to define acceptance can be worded as follows: (i) who is impacted the most by the consequences of human-wildlife issues? and (ii) should you consider people as accepting a consequence if they have no interest or stake in the conflict about wildlife?

\section{CONCLUSIONS}

It is clear from the two workshops that participants have different perspectives on how to define tolerance, acceptance, and coexistence. The authors of this paper respect this output and believe that we can coexist with this diversity of ideas and continue to work toward deepening our understanding of the concepts.

Regardless of definition, there does appear to be a commonly recognized hierarchy from tolerance to acceptance to coexistence. Furthermore, there is some consensus about the level at which the concepts are defined and operationalized: tolerance and acceptance describe individual attitudes and behaviors, and coexistence is more broadly nested in the socialecological landscape. Future research should continue to explore the relationships between these concepts and at which scale they are applied. 
Given the complexity and variability of perspectives about coexistence, we recommend that researchers, managers and decisions-makers engage in inductive inquiry that avoids unspoken and untested assumptions about human-wildlife interactions. Definitions should not be pre-determined without community and stakeholder input, especially when researchers/managers and decision-makers are new to an area, community, or project, and may hold different perspectives than those involved in the human-wildlife interactions. We suggest considering the specific context and needs, and then identifying and clearly articulating relevant concepts-regardless of their names-that can then be measured. Similarly, we emphasize that there is a need to develop a glossary of terms when embarking on a research project. Such development will help with clarify definitions among research teams, lead to a more robust understanding of how to appropriately measure tolerance, acceptance, and coexistence, and enable shared interpretation of findings. We believe this will ultimately assist with further development in the scholarly literature around these terms and allow academics to continue to deliberate, debate, and progress toward a more unified set of definitions and measurements.

\section{DATA AVAILABILITY STATEMENT}

The raw data supporting the conclusions of this article will be made available by the authors, without undue reservation.

\section{REFERENCES}

Brenner, L. J., and Metcalf, E. C. (2020). Beyond the tolerance/intolerance dichotomy: incorporating attitudes and acceptability into a robust definition of social tolerance of wildlife. Hum. Dimens. Wildl. 25, 259-267. doi: 10.1080/10871209.2019.1702741

Bruskotter, J. T., Singh, A., Fulton, D. C., and Slagle, K. (2015). Assessing tolerance for wildlife: clarifying relations between concepts and measures. Hum. Dimens. Wildl. 20, 255-270. doi: 10.1080/10871209.2015.1016387

Bruskotter, J. T., and Wilson, R. S. (2014). Determining where the wild things will be: using psychological theory to find tolerance for large carnivores. Conserv. Lett. 7, 158-165. doi: 10.1111/conl.12072

Carter, N. H., and Linnell, J. D. C. (2016). Co-adaptation is key to coexisting with large carnivores. Trends Ecol. Evol. 31, 575-578. doi: 10.1016/j.tree.2016.05.006

Chapron, G., and López-Bao, J. V. (2016). Coexistence with large carnivores informed by community ecology. Trends Ecol. Evol. 31, 578-580. doi: 10.1016/j.tree.2016.06.003

Crespin, S. J., and Simonetti, J. A. (2019). Reconciling farming and wild nature: integrating human - wildlife coexistence into the land-sharing and landsparing framework. Ambio 48: 131-138. doi: 10.1007/s13280-018-1059-2

Frank, B. (2016). Human-wildlife conflicts and the need to include tolerance and coexistence: An introductory comment. Soc. Nat. Resour. 29, 738-743. doi: 10.1080/08941920.2015.1103388

Frank, B., and Glikman, J. A. (2019). Human-Wildlife Conflicts and the Need to Include Coexistence. Chapter 1 pages 1-19, IN: Human-Wildlife Interactions: Turning Conflict into Coexistence, Vol. 23. Cambridge: Cambridge University Press.

Frank, B., Glikman, J. A., and Marchini, S. Eds. (2019). Human-Wildlife Interactions: Turning Conflict Into Coexistence, Vol. 23. Cambridge: Cambridge University Press.

\section{ETHICS STATEMENT}

The studies involving human participants were reviewed and approved through the Institutional Review Board (IRB\# 03849e) of Miami University, Ohio, USA. Written informed consent for participation was waived due to the conference context. This is in accordance with the national legislation and the institutional requirements.

\section{AUTHOR CONTRIBUTIONS}

JG, BF, and KR contributed to design of the study methods. KR facilitated the World Café in Namibia whereas JG and AM facilitated the World Café in Toronto. JK organized the results. JG, KR, and JK wrote the first draft of the results section. BF and JG wrote the first draft of introduction. CS and EM wrote the first draft of the discussion. SM wrote sections across all manuscript. All authors contributed to several discussions on the conception of the manuscript, contributed to manuscript, revision, read, and approved the submitted version.

\section{FUNDING}

At the time of developing and carrying out the World Café, JG was the associate director of the Community Engagement Team of the San Diego Zoo's Institute for Conservation Research. JK was supported by a research fellowship from Institute for Conservation Research.

Glikman, J. A., Frank, B., and Marchini, S. (2019). Human-Wildlife Interactions. Multifaceted Approaches for Turning Conflict into Coexistence. Chapter 20 page 439- 452, IN: Human-Wildlife Interactions: Turning Conflict into Coexistence. Vol. 23. Cambridge: Cambridge University Press.

Hazzah, L., Chandra, S., and Dolrenry, S. (2019). Leaping Forward: The Need for Innovation in Wildlife Conservation. chapter 17 page 359- 383. in: HumanWildlife Interactions: Turning Conflict into Coexistence, Vol. 23. Cambridge: Cambridge University Press.

Holling, C. S. (2001). Understanding the complexity of economic, ecological, and social systems. Ecosystems 4, 390-405. doi: 10.1007/s10021-001-0101-5

IUCN (2020). IUCN SSC Position Statement on the Management of Human-Wildlife Conflict. IUCN Species Survival Commission (SSC) Human-Wildlife Conflict Task Force. Available online at: www.iucn.org/theme/species/publications/policies-and-position-statements (accessed August 27, 2021).

Johnson, H. E., Lewis, D. L., Lischka, S. A., and Breck, S. W. (2018). Assessing ecological and social outcomes of a bear-proofing experiment. J. Wildl. Manag. 82, 1102-1114. doi: 10.1002/jwmg.21472

Kansky, R., Kidd, M., and Fischer, J. (2021). Does money "buy" tolerance toward damage-causing wildlife? Conserv. Sci. Practice 3:e262. doi: 10.1111/csp2.262

Kansky, R., Kidd, M., and Knight, A. T. (2016). A wildlife tolerance model and case study for understanding human wildlife conflicts. Biol. Conserv. 201, 137-145. doi: 10.1016/j.biocon.2016.07.002

Knox, J., Ruppert, K., Frank, B., Sponarski, C. C., and Glikman, J. A. (2021). Usage, definition, and measurement of coexistence, tolerance and acceptance in wildlife conservation research in Africa. Ambio 50, 301-313. doi: 10.1007/s13280-020-01352-6

König, H. J., Kiffner, C., Kramer-Schadt, S., Fürst, C., Keuling, O., and Ford, A. T. (2020). Human-wildlife coexistence in a changing world. Conserv. Biol. 34, 786-794. doi: 10.1111/cobi.13513 
Lamb, C. T., Ford, A. T., McLellan, B. N., Proctor, M. F., Mowate, G., Ciarniello, L., Nielsen, S. E., and Boutin, S. (2020). The ecology of humancarnivore coexistence. Proc. Natl. Acad Sci. U. S. A. 117, 17876-17883. doi: 10.1073/pnas.1922097117

Lischka, S. A., Teel, T. L., Johnsond, H. E., and Crooks, K. R. (2019). Understanding and managing human tolerance for a large carnivore in a residential system. Biol. Conserv. 238: 108189. doi: 10.1016/j.biocon.2019.07.034

Lute, M. L., and Carter, N. H. (2020). Are we coexisting with carnivores in the American West? Front. Ecol. Evol. 8, 1-13. doi: 10.3389/fevo.2020.00048

Morehouse, A. T., and Boyce, M. S. (2017). Troublemaking carnivores: Conflicts with humans in a diverse assemblage of large carnivores. Ecol. Soc. 22, 4. doi: 10.5751/ES-09415-220304

Pooley, S., Barua, M., Beinart, W., Dickman, A., Holmes, G., Lorimer, J., and Milner-Gulland, E. J. (2017). An interdisciplinary review of current and future approaches to improving human-predator relations. Conserv. Biol. 31, 513-523. doi: 10.1111/cobi.12859

Pooley, S., Bhatia, S., and Vasava, A. (2020). Rethinking the study of humanwildlife coexistence. Conserv Biol. 35, 784-793. doi: 10.1111/cobi.13653

Redpath, S. M., Bhatia, S., and Young, J. (2015). Tilting at wildlife: reconsidering human-wildlife conflict. Oryx 49, 222-225. doi: 10.1017/S0030605314000799

Slagle, K., and Bruskotter, J. T. (2019). Tolerance for Wildlife: A Psychological Perspective. chapter 5 page 85-106. in: Human-Wildlife Interactions: Turning Conflict into Coexistence. Vol. 23. Cambridge: Cambridge University Press.

The World Café, Community Foundation Creative Commons, Attribution (2015). A Quick Reference Guide for Hosting World Café. Available online at: www.theworldcafe.com (accessed September 7, 2021).

Treves, A., and Santiago-Ávila, F. J. (2020). Myths and assumptions about human- wildlife conflict and coexistence. Conserv. Biol. 34, 811-818. doi: $10.1111 /$ cobi.13472 van Eeden, L., Dickman, C., Crowther, M., and Newsome, T. (2021). A Theory of Change for promoting coexistence between dingoes and livestock production. Conserv. Sci. Practice 3:e304. doi: 10.1111/ csp2.304

Woodroffe, R., Thirgood, S., and Rabinowitz, A. (eds.). (2005). People and Wildlife, Conflict or Co-Existence?. Cambridge: Cambridge University Press.

Yurco, K., King, B., Young, K. R., and Crews, K. A. (2017). Humanwildlife interactions and environmental dynamics in the Okavango Delta, Botswana. Soc. Nat. Resour. 30, 1112-1126. doi: 10.1080/08941920.2017. 1315655

Conflict of Interest: The authors declare that the research was conducted in the absence of any commercial or financial relationships that could be construed as a potential conflict of interest.

Publisher's Note: All claims expressed in this article are solely those of the authors and do not necessarily represent those of their affiliated organizations, or those of the publisher, the editors and the reviewers. Any product that may be evaluated in this article, or claim that may be made by its manufacturer, is not guaranteed or endorsed by the publisher.

Copyright (C) 2021 Glikman, Frank, Ruppert, Knox, Sponarski, Metcalf, Metcalf and Marchini. This is an open-access article distributed under the terms of the Creative Commons Attribution License (CC BY). The use, distribution or reproduction in other forums is permitted, provided the original author(s) and the copyright owner(s) are credited and that the original publication in this journal is cited, in accordance with accepted academic practice. No use, distribution or reproduction is permitted which does not comply with these terms. 